\title{
Using Geolocation Information for Dynamic Spectrum Access in Cellular Networks
}

\author{
Jad Nasreddine* $\quad$ Natalia Miliou ${ }^{\dagger} \quad$ Janne Riihijärvi* \\ Andreas Polydoros ${ }^{\dagger} \quad$ Petri Mähönen* \\ *Institute for Networked Systems (iNETS) \\ RWTH Aachen University \\ Aachen, Germany \\ † Institute of Accelerating Systems and Applications (IASA) \\ National Kapodistrian University of Athens (NKUA) \\ Athens, Greece \\ \{jad,jar,pma\}@inets.rwth-aachen.de,\{nmiliou,polydoros\}@phys.uoa.gr
}

\begin{abstract}
In this paper, we explore the possibility of deploying a cognitive network that can opportunistically access the spectrum allocated to a primary cellular network. The main problem is that cellular networks are characterized by multiple simultaneous and dynamic transmissions combined with power control. The proposed approach assumes that the cognitive network knows the positions of the primary base stations and its own base stations. It utilizes a Maximum Likelihood Estimator (MLE) to detect the activity of neighboring primary base stations and exploits this information to determine the power with which a cognitive radio can transmit. The results show the possibility of using such a method to deploy an IEEE 802.11-like network as a cognitive network under specific constraints related to the position, the number and the distribution of the spectrum monitoring sensors inside the primary network.
\end{abstract}

\section{Categories and Subject Descriptors}

G.3 [Probability and Statistics]: Statistical computing; I.6.4 [Simulation and Modeling]: Model Validation and Analysis; I.6.5 [Simulation and Modeling]: Model Development; I.6.6 [Simulation and Modeling]: Simulation Output Analysis

\section{General Terms \\ Performance, Algorithms, Theory}

Permission to make digital or hard copies of all or part of this work for personal or classroom use is granted without fee provided that copies are not made or distributed for profit or commercial advantage and that copies bear this notice and the full citation on the first page. To copy otherwise, to republish, to post on servers or to redistribute to lists, requires prior specific permission and/or a fee.

$P M^{2} H W^{2} N^{\prime} 11$, October 31, 2011, Miami, Florida, USA.

Copyright 2011 ACM 978-1-4503-0902-8/11/11 ...\$10.00.

\section{Keywords}

Maximum Likelihood Estimator; geolocation; dynamic spectrum access; cognitive radio.

\section{INTRODUCTION}

The last decade has witnessed a fast evolution of wireless communication networks from physical to application layers. Cognitive Radio (CR) and Dynamic Spectrum Access (DSA) are among the most promising new developed concepts. These concepts are proposed to solve the problem of spectrum usage efficiency by implementing more flexible frequency allocation approaches $[5,8,17]$. In this context, several techniques have been developed to allow secondary users or cognitive radios to access a licensed spectrum in an opportunistic way. Opportunistic access should satisfy a set of rules imposed by the spectrum licensee or primary network, such as interference probability $[13,15]$.

Most of the work in DSA framework focuses on TV white space where the primary network is a TV broadcasting network $[7,12,13,17]$. Although TV white space offers a huge opportunity to increase spectrum usage efficiency, other possibilities are still understudied. One of these possibilities is to allow DSA in systems where the primary network is a cellular network. Of course, spectrum opportunities in this case are more difficult to detect, but they offer additional possibilities especially since more frequencies are now allocated to this type of networks [3,4]. This will be possible in the future especially if spectrum regulation policies change and flexible approaches such as DIMSUMnet [6] are implemented. In this case, some frequency bands can be allocated to dominating operators, as a primary network, with the possibility of sharing these bands with other operators or service providers, which act as secondary networks. This is especially possible if (1) those operators do not provide the same service, (2) the price paid for these bands by the primary operator is lower than the price of other bands, and (3) a conservative approach towards protecting primary users is adopted by secondary networks. Machine to Machine (M2M) communication and monitoring networks are possible candidates of such secondary networks. 


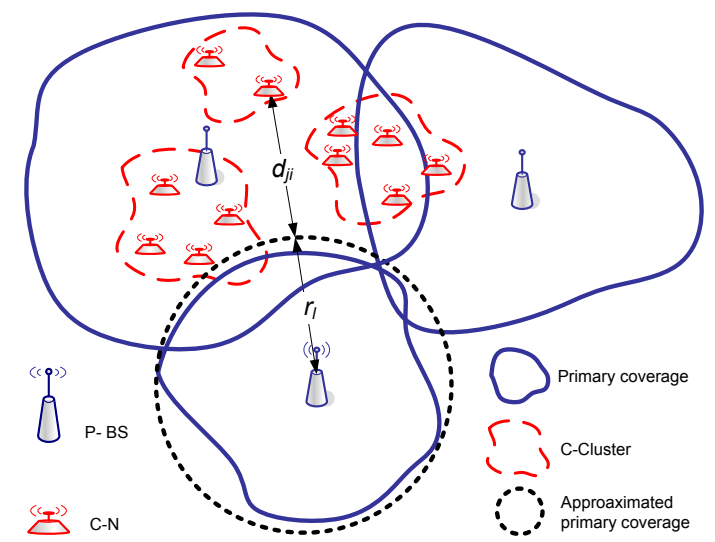

Figure 1: Primary and secondary networks.

Detecting spectrum opportunities in the frequency bands allocated to cellular networks is more challenging than in $\mathrm{TV}$ white space due to the challenging characteristics of the former, namely the requirement for pervasive coverage, the presence of different services with different quality constraints, dynamic traffic patterns, adaptive primary transmit powers, and the presence of several neighboring primary transmitters. The first two problems can be normally dealt with by defining suitable interference constraints such as in [14]. Primary traffic dynamics are taken into account by using fast and cooperative sensing techniques [5]. The problems of multiple transmitters and dynamic transmit power are starting to attract researchers and several approaches have been presented in the last few years $[11,13,15]$. These proposals consider either the most difficult case where no information about the power and the location of Primary Base Stations (P-BSs) is known $[11,13]$ or the simple case where both location and transmit power are known [15] by the CR. In some countries the locations of cellular base stations can be available for public based on regulatory demand [1]. However, transmit power can change very fast, especially if fast power control or fast scheduling is applied. Hence, a reasonable assumption in the case of primary cellular network is to consider that the CR knows the positions of the $\mathrm{P}-\mathrm{BSs}$ through a local or regional database as it is suggested by the FCC [2] without knowing their transmit powers.

In this paper we study the possibility of employing a Maximum Likelihood Estimator (MLE) to detect the activity of primary base stations and exploit this information to determine the power with which a cognitive node can transmit knowing its own position and the positions of primary base stations. The limitations of this approach are also studied.

The paper is organized as follows. In Section 2, we present the system model and we formulate the problem of finding the allowed transmit power of cognitive nodes. In Section 3, we show how the MLE technique can be used to identify the active base stations. In Section 4, simulation results are provided and analyzed. In Section 5, we conclude the paper and highlight some possible future works.

\section{SYSTEM MODEL AND PROBLEM FORMULATION}

Consider a $M$ cognitive radio clusters (C-Clusters) deployed in the coverage area of a cellular network as shown in Figure 1. A C-Cluster $c$ is formed by set $\mathcal{S}_{c}$ of cooperative Cognitive Nodes (C-Ns) that act also as spectrum monitoring sensors. The locations of the $\mathrm{C}-\mathrm{Ns}$ follow a probability distribution $\mathcal{D}$. The primary network involves $N$ base stations that can be either active or idle in a given channel. The activities of these base stations are supposed to be unknown to the C-Ns. In particular, the transmit power $P_{l}$ of an active primary transmitter $l$ is not known. This is realistic assumption since base station activities in cellular networks can have very high dynamics. Therefore, unacceptable signaling traffic will be generated if the activity information is to be sent to the C-Ns in real time.

Without loss of generality, we consider only one channel used by the primary network in our analysis. In the following we denote by $\Phi$ the set of all primary base stations and by $\Phi_{a}$ the set of primary active base stations using the channel of interest. The objective of our proposed DSA approach is to determine the power with which a $\mathrm{C}-\mathrm{N}$ can transmit, while respecting primary constraints, based on the measured power on the channel of interest and its location. For clarity, we use the following notations: $j \in\left\{1,2, \ldots, \sum_{1}^{M}\left|\mathcal{S}_{c}\right|\right\}$ to denote a cognitive node, and $l \in\left\{\sum_{1}^{M}\left|\mathcal{S}_{c}\right|+1, \sum_{1}^{M}\left|\mathcal{S}_{c}\right|+\right.$ $\left.2, \ldots, \sum_{1}^{M}\left|\mathcal{S}_{c}\right|+N\right\}$ to denote a primary base station, where $\left|\mathcal{S}_{c}\right|$ is the cardinality of set $\mathcal{S}_{c}$. Moreover, index $i$ denoting a receiver in a primary cell has the same range of index $l$. In addition, spectrum monitoring sensors are referred to as sensors in the rest of the paper.

As in all cellular networks, a transmitter can be either idle or active with power ranging from $P_{\min }$ to $P_{\max }$. These bounds are known by the cognitive network since this information is specific for the used technology and defined by standardization bodies. The same restriction is applied on the C-Ns that should transmit using power higher than $P_{\text {th }}$ due to hardware constraints. If the computed allowed power is less than $P_{\mathrm{th}}$, the C-N cannot transmit at all.

\subsection{Primary Constraint}

In this paper, we assume that the primary constraint is a limit on the interference probability experienced by any primary receiver $i[13,15]$. This constraint is defined by

$$
\mathbb{P}\left\{I_{i}>\iota_{\max }\right\} \leq \varepsilon,
$$

where $I_{i}$ is the experienced interference by receiver $i$ due to secondary activity, $\iota_{\max }$ is the interference threshold and $\varepsilon$ is the interference probability threshold, defined by the primary network. We also assume that all C-Ns have access to a central database that distributes the frequency bands, and thus only one $\mathrm{C}-\mathrm{N}$ can transmit at a given channel and time. Although this type of allocation is rather difficult to implement in operational systems, the determination of the transmit power in a multiple secondary transmitter context is still an open problem and several methods are proposed to solve it $[7,9]$. Hence, we do not consider this problem in this paper, but the model can be easily extended to the general case. By considering this assumption, $I_{i}$ becomes the result of the transmission of one $\mathrm{C}-\mathrm{N}$ and can be computed as

$$
I_{i}=P_{j} G_{j i}
$$

where $P_{j}$ is the secondary transmit power and $G_{j i}$ is the path gain between nodes $i$ and $j$ given by

$$
G_{j i}=\frac{k_{s p}}{d_{j i}^{\alpha_{s p}}} \xi_{j i},
$$




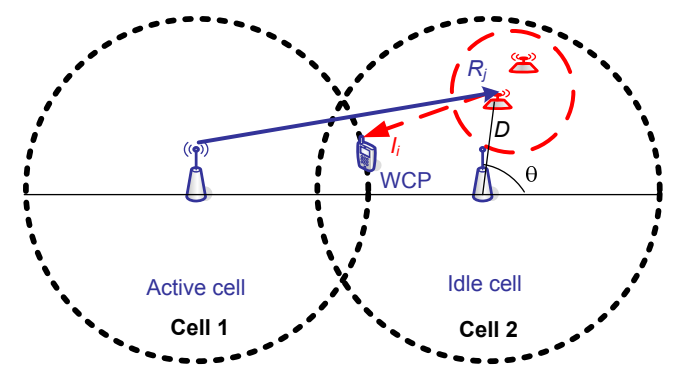

Figure 2: An illustrative example of the model with two cells.

where $d_{j i}$ is the distance separating the two nodes, $k_{s p}$ and $\alpha_{s p}$ are propagation constants that depend on the type of the nodes, and $\xi_{j i} \sim \ln \mathcal{N}\left(0, \sigma_{s p}^{2}\right)$ is a log-normally distributed random variable representing the shadowing effect.

From (1), (2) and (3), $P_{j}$ can be written as

$$
P_{j} \leq \frac{\iota_{\max } d_{j i}^{\alpha_{s p}}}{k_{s p}} e^{\sqrt{2} \sigma_{s p} \operatorname{erf}^{-1}(2 \varepsilon-1)} .
$$

\subsection{Problem Formulation}

As shown in the previous paragraph, the computation of $P_{j}$ requires the knowledge of the distance between the C-N and the closest active primary receiver or at least a lower bound on this distance. If the primary network is active in uplink, $d_{j i}$ can be computed accurately since the position of the receiver (i.e., base station) is known. If the primary network is active in downlink however, only the lower bound of the distance can be estimated since the position of the receiver (i.e., terminal) cannot be known accurately. Hence, we assume that the coverage area of the primary network is known and it is approximated by the disc with radius $R_{i}$ encompassing the real one (See Figure 1). In this case, the lower bound is computed as the distance between the transmitting $\mathrm{C}-\mathrm{N}$ and the closest point inside the disc. Here two cases appear: if the $\mathrm{C}-\mathrm{N}$ is inside the coverage area, the lower bound becomes 0 since the primary receiver can be at any location inside the coverage area. If the $\mathrm{C}-\mathrm{N}$ is outside the coverage area, the lower bound is the distance between the $\mathrm{C}-\mathrm{N}$ and the closest point or worst case position (WCP) at the borders of the covering disc as shown in Figure $2[15,16]$. To detect the closest active receiver, the closest active primary cell (i.e., where a base station or a terminal is transmitting) has to be detected first. The main problem in detecting this cell is the presence of multiple simultaneous primary transmitters. In the following, we consider the downlink case and the model can be easily extended to the uplink case. In this case, each sensor $j$ receives power $R_{j}$, which is the aggregation of the signals received from the active primary base stations

$$
\begin{aligned}
R_{j} & =\sum_{l \in \Phi} R_{l j} \\
& =\sum_{l \in \Phi} P_{l} G_{l j},
\end{aligned}
$$

where $P_{l} \in\{0\} \cup\left[P_{\min }, P_{\max }\right]$ is the primary transmit power and $G_{l j}$ is the path gain between P-BS $l$ and C-N $j$

$$
G_{l j}=\frac{k_{p s}}{d_{l j}^{\alpha_{p s}}} \xi_{l j}
$$

One of the difficulties to detect the active P-BSs based on the received power is the presence of the shadowing factor $\xi_{l j} \sim \ln \mathcal{N}\left(0, \sigma_{p s}^{2}\right)$ between P-BSs and C-Ns. In the next section we show how we can detect these base stations using a Maximum Likelihood Estimator (MLE).

\section{COOPERATIVE DSA BASED ON GEOLOCATION INFORMATION}

To detect their closest active cells, all C-Ns in each set $\mathcal{S}_{c}$ exchange the measured received signals or send them to a central unit. The probabilistic description of $R_{j}$ when multiple primary transmitters are active (i.e. $P_{l}$ is non-zero for multiple values of $l$ ) has been studied in [11]. In particular, it was shown that the distribution of $R_{j}$ can be approximated by the $\log$-normal distribution $\ln \mathcal{N}\left(\mu_{j}, \beta_{j}\right)$ where

$$
\mu_{j}=\ln \left(\sum_{l \in \Phi} \frac{k_{p s} P_{l}}{d_{l j}^{\alpha p s}}\right)+\frac{\sigma_{p s}^{2}}{2}-\frac{\beta_{j}}{2}
$$

and

$$
\beta_{j}=\ln \left[\left(e^{\sigma_{p s}^{2}}-1\right) \frac{\sum_{l \in \Phi}\left(\frac{k_{p s} P_{l}}{\left.d_{l j}^{\alpha}\right)^{\alpha}}\right)^{2}}{\left(\sum_{l \in \Phi} \frac{k_{p s} P_{l}}{d_{l j}^{\alpha} p s}\right)^{2}}\right] .
$$

Since we assume that the locations of the base stations are known, these expressions relate the total received power as a log-normal random variable directly to the set of active base stations and their transmit powers. We can therefore apply the maximum likelihood criterion to estimate the activities of the base stations, and subsequently to estimate the allowable transmit power of the $\mathrm{C}-\mathrm{N}$ based on the state of the primary network. Finding the MLE yields a non-convex optimization problem which is difficult to handle analytically, especially for large number of P-BSs. There exist multiple ways to approach this problem numerically. In this work, we apply a numerical approach based on simulated annealing to solve the arising maximum likelihood estimation problem. In [11], simulations were performed based on the simulated annealing technique and the performance of the estimator was investigated as function of various channel parameters (e.g., fading statistics, path loss parameter) and the number of sensors. It was shown that under realistic assumptions for the channel, the sources and the sensors the algorithm succeeds to estimate the transmitters' activity.

In this work, we are interested only in knowing if the P-BS is active or not (i.e., determining set $\phi_{a}$ ) and not in determining $P_{l}$, which facilitates the decision and reduces the error in the estimation of the closest active P-BS. When set $\phi_{a}$ is determined, each C-N determines its closest cell from this set and determines its allowed power based on (4). If the allowed power is higher than $P_{\text {th }}$, the C-N can transmit. Otherwise it will stay idle or choose another channel.

\section{SIMULATIONS AND RESULTS}

To study the performance of the proposed approach and analyze its limitations, simulations in Matlab were conducted.

\subsection{Simulation Model}

We evaluate our approach in a system where the primary network is a cellular network consisting of only macrocells served by base stations at a height of $27 \mathrm{~m}$. These P-BSs 
serve mobile terminals (M-Ts) with antenna height of $1.5 \mathrm{~m}$. The C-Ns are considered to be IEEE 802.11 kind of access points (i.e., modified access points to enable cognitive features) with antenna height of $3 \mathrm{~m}$. In all simulations we consider the downlink of the primary and secondary networks, i.e., the transmitters are the P-BSs and the C-Ns.

We use the Xia-Bertoni propagation model [10]. This model is chosen since it is able to take into account all types of propagation losses such as the one between base stations and the one between mobiles in addition to usual propagation loss between a base station and a mobile. Given a frequency $f$ in $\mathrm{GHz}$ and distance $d_{X Y}$ between transmitter $X$ and receiver $Y$, path gain $G_{X Y}$ is given by

$$
G_{X Y}\left(d_{X Y}\right)=K_{X Y}+\beta_{X Y} \log _{10}(f)+\alpha_{X Y} \log _{10}\left(d_{X Y}\right),
$$

where $K_{X Y}, \beta_{X Y}$ and $\alpha_{X Y}$ are constants computed using the Xia-Bertoni model as depicted in Table 1.

\subsection{Scenario with Two Cells}

First, we shall study the performance of the proposed algorithm as function of the number of sensors in a simple scenario with two cells of $1 \mathrm{~km}$ radius. For this we consider that $P_{\max }=27.4 \mathrm{dBm}, P_{\min }=17.4 \mathrm{dBm}$ and $P_{\mathrm{th}}=20 \mathrm{dBm}$. Therefore if the $\mathrm{C}-\mathrm{N}$ determines that the primary transmit power is lower than $17.4 \mathrm{dBm}$, it will identify the corresponding base station as idle. Regarding the standard deviation of the shadowing between the P-BS and C-N, we use $\sigma_{p s}=1.38$ (this corresponds to $6 \mathrm{~dB}$ ) except for the case where the impact of $\sigma_{p s}$ is studied. Simulations are repeated 200 times for each setting in order to compute the marginal distributions. In the simulations we consider the following three scenarios:

- Scenario 1: One base station (i.e., cell 1 in Figure 2) is active while the second is idle (i.e., cell 2 in Figure 2). The sensors are distributed uniformly inside one cell.

- Scenario 2: The two base stations are active. The sensors are distributed uniformly inside one cell.

- Scenario 3: The activity pattern is the same as in scenario 1, but the sensors are distributed uniformly inside the two cells.

By considering that the primary transmit power is $24 \mathrm{dBm}$, we show in Figure 3 the cumulative distribution functions (cdfs) of the estimated power of the primary base stations, for different numbers of sensors and considering the three scenarios. It should be noted that the lines that do not appear in the figure correspond to the cases where the C-Ns are not allowed to transmit. In scenario 1, the figure shows that with 17 sensors or more this base station is detected to be active in more than $90 \%$ of the cases. However, this percentage drops to $60 \%$ in case only one sensor is in the active cell and to $40 \%$ when the sensor is in the idle cell. This shows the importance of cooperative detection and that the proposed method can protect the primary receivers if the number of sensors is large enough. Furthermore, the figure shows that the inactivity of the idle base station (i.e., spectrum opportunity) can be detected with high accuracy when the sensors are in the idle cell. Even in the case of one sensor, a false detection appears in less than $5 \%$ of the studied cases. However, when the sensors are in the active cell the false detection increase to $60 \%$ in case we have more than 9 sensors and to more than $95 \%$ in case we have only one sensor. This shows how many opportunities can be lost
Table 1: Constants of the propagation model.

\begin{tabular}{lllll}
\hline & $\alpha$ & $\beta$ & $k$ & $\sigma$ \\
\hline $\mathrm{C}-\mathrm{N} \leftrightarrow \mathrm{P}-\mathrm{BS}$ & -37.6 & -21 & -113.2 & {$[0.69 ; 3.45]$} \\
$\mathrm{C}-\mathrm{N} \leftrightarrow \mathrm{M}-\mathrm{T}$ & -40 & -30 & -141.7 & 2.3 \\
\hline
\end{tabular}

due to the position of the sensors. This is normal due to the high shadowing standard deviation. For example, a sensor situated at the common boundary of two equally sized cells, with both primary transmitters utilizing the same transmit powers, would have to estimate whether one or both transmitters are active based on expected difference in average received power of approximately $3 \mathrm{~dB}$. However, given that the typical standard deviation of the shadowing component is higher than $3 \mathrm{~dB}$, we see that the differences in the activation patterns are easily overshadowed by the contributions from shadowing in terms of likelihoods. This effect can be eliminated by distributing the sensors over the two cells as it shown in the plots corresponding to scenario 3. Moreover the plots corresponding to scenario 2 show that the sensors can detect the activity of the two cells with high probability. However, the level of the power of the non covering cell is significantly reduced due to the high separating distance. In summary, these results show that the positions of the sensors can have more impact that their number.

In Figure 4, we show the false alarm probability (i.e, the probability that the idle base station is considered active) and the miss-detection probability (i.e., the probability that the active base station is considered idle). In this figure, the previously discussed effect becomes more clear. In addition, the impact of the primary transmit power is shown, where the false alarm is an increasing function whereas the missdetection is a decreasing function of this power. This means that the higher the latter is, the more protective will be the algorithm. Moreover, this figure shows that the number of sensors required to protect the primary (i.e., keep the miss-detection probability lower than a threshold) decreases when the primary transmit power increases but this increase in power will decrease the possibility of detecting spectrum opportunities. Furthermore, when the power is very low, e.g., $17 \mathrm{dBm}$, the primary receivers cannot be protected with any number of sensors. In Figure 5, we show the cdf of the allowed transmit power in the three scenarios as a function of the number of sensors when the primary power is $24 \mathrm{dBm}$. The results of this figure reflect the ones shown in Figure 3 from the secondary network's point of view. We only highlight here that in most of the cases where the C-Ns are close to the idle base stations the secondary transmit power is relatively high.

In Figure 6, we show the distribution of the estimated allowed power for different values of $\sigma_{p s}$ (i.e., the standard deviation of the shadowing factor between P-BS and C-N) in scenario 1 where the primary power is $24 \mathrm{dBm}$ and there are 17 sensors. The figure shows that the estimated power decreases when $\sigma_{p s}$ increases. Moreover, the miss-detection probability that can be estimated from the left-hand figure (i.e., the probability that the power is higher than $20 \mathrm{dBm}$ ) increases with the standard deviation, which reduces the protection of the primary network. However, for a standard deviation of 2 , which is a typical value for urban zones, the miss-detection probability is less than 0.05 . 

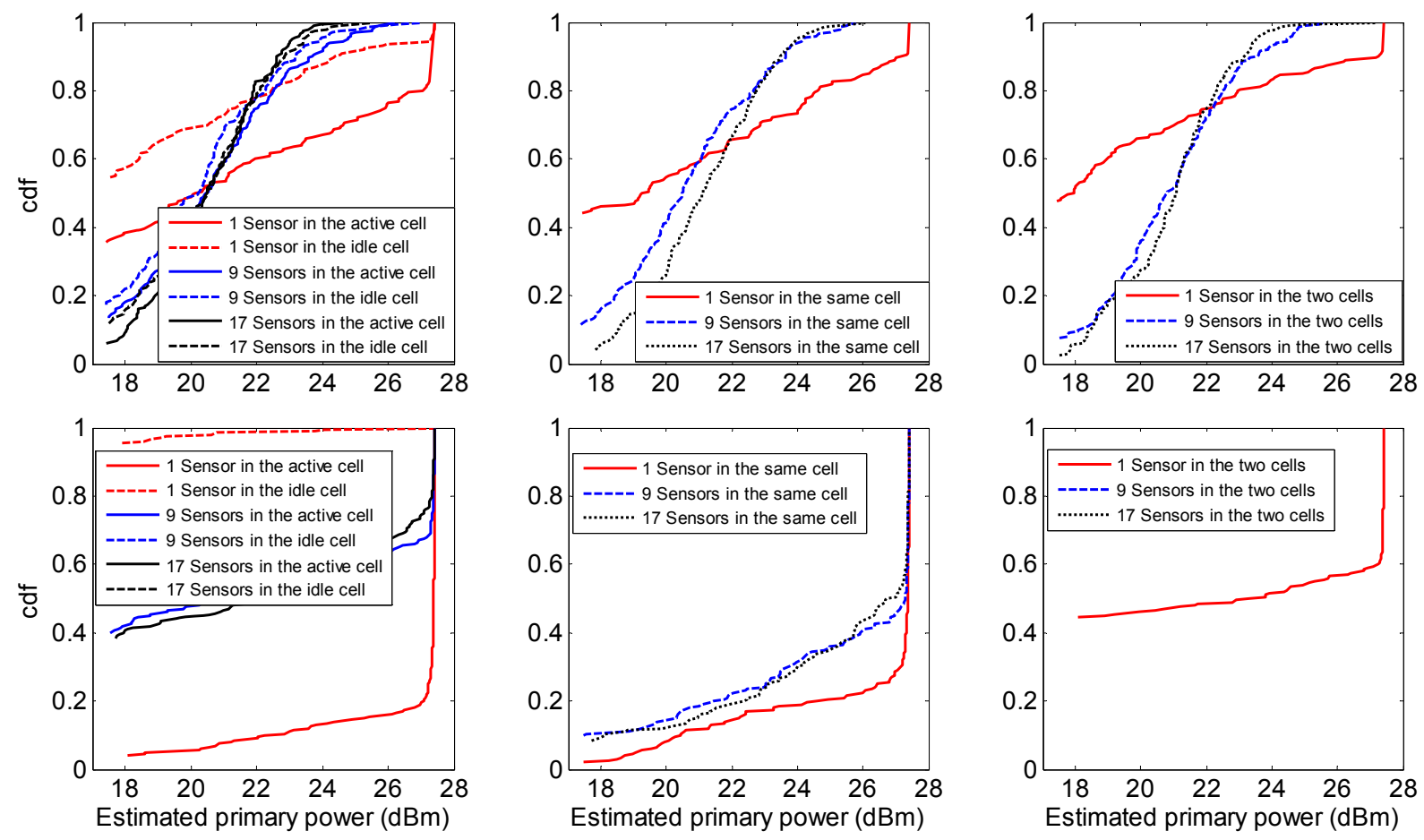

Figure 3: The distribution of the estimated power of primary base stations by secondary network. The first (resp. the second) row reflects the estimated power of cell 1 (resp. cell 2) of Figure 2, while the three columns reflect the three simple scenarios. The legend "One sensor in the two cells" in scenario 3 means that the sensor position is uniformly distributed over the two cells.
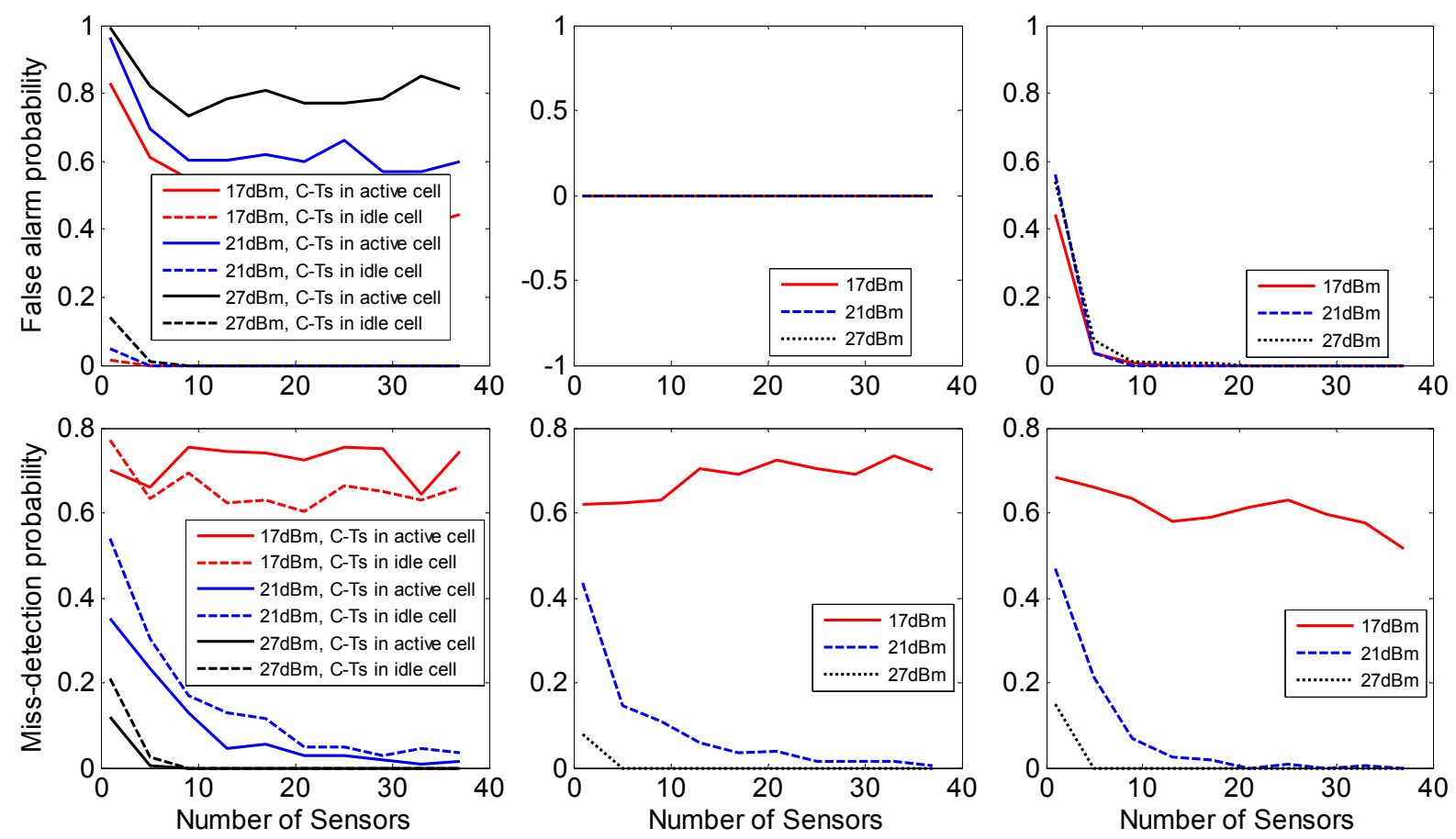

Figure 4: The variation of the false alarm probability (first row) and miss-detection probability (second row) as a function of the number of sensors for different transmit power levels of the primary transmission. The three columns reflect the three simple scenarios. 

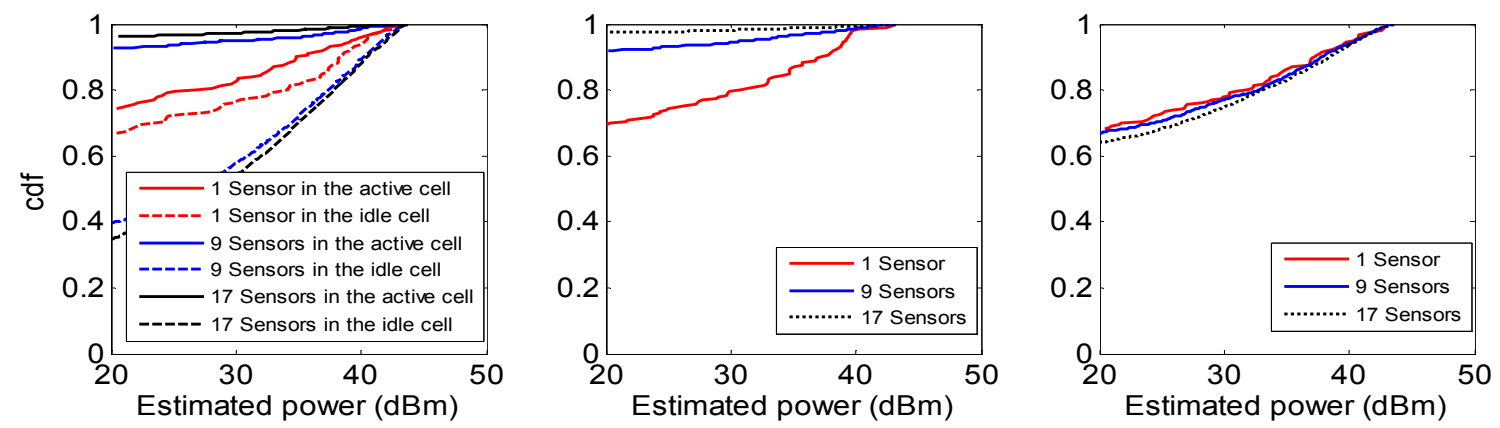

Figure 5: The distribution of the estimated power with which the secondary users can transmit for different numbers of sensors.
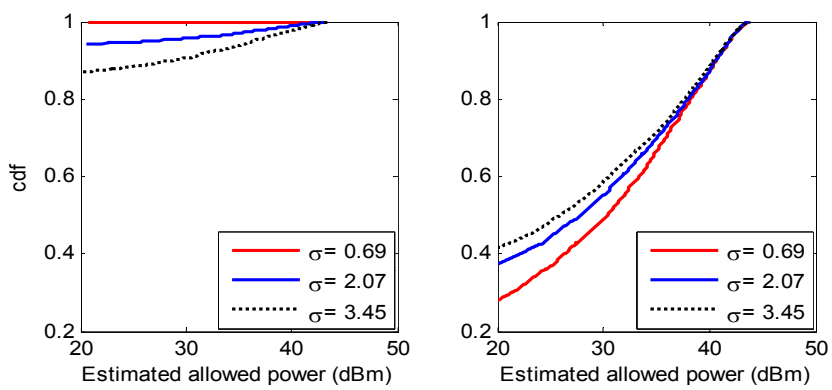

Figure 6: The distribution of the estimated allowed power for different values of $\sigma_{p s}$ in scenario 1 , when the sensors are in the active cell (figure on the left) and in the idle cell (figure on the right).

In order to consider the more general case, we assume now that the C-Ns are distributed inside a cluster that is not centered at the P-BS. Scenario 1 is considered here where the primary power is $24 \mathrm{dBm}$ and there are 17 sensors. Figure 7 shows the impact of the position and the size of the cluster on C-N's transmit power when the sensors are inside the idle cell. As expected, the power increases as the size of the clusters increases since the sensors are distributed over a larger surface in this case, yielding less correlation in the received signals. Furthermore, the cdf of the power starts at higher values for smaller clusters since the minimum distance separating the C-Ns from the active P-BS is higher than when bigger clusters are used. Moreover, this power increases when the cluster is closer to the idle cell. It can be noted that when the cluster is between the two base stations (i.e., the angle $\theta$ of Figure 2 is equal to $180^{\circ}$ ), the secondary opportunities to transmit and the allowed transmit power decrease drastically especially for small clusters (e.g., no transmission is allowed if the cluster radius is less than $0.22 \mathrm{~km})$.

It should be noted that the miss-detection of the active base station does not always yield a harmful interference for primary users since we are considering a protective scheme. In fact, we evaluated the probability of harmful interference by generating one primary receiver in each active cell, where the position of these receivers are uniformly distributed inside the cell. The results have shown that except from the case of one sensor, the probability of harmful interference was always lower than 0.05 .

\subsection{Los Angeles Scenario}

We shall next study the distribution of the transmit powers for the C-Ns running in a more realistic scenario where the base stations are distributed in a selected area of $400 \mathrm{~km}$ in Los Angeles city based on the location data from the TMobile network [1]. We consider that three frequency bands are available for the primary network. The distribution of the frequency bands over the base stations is optimized in order to minimize the total received interference in the system, where each base station can be associated to one band. The coverage areas of the base stations are optimized in order to have a full coverage in the studied area. Moreover, we consider that the maximum transmit power of each base station is computed so that users at the border of the coverage area have an SNR higher than $1.5 \mathrm{~dB}$ in $95 \%$ of the cases. In order to evaluate the proposed method we consider two cells that are using frequency band 1 as shown in Figure 8. These cells have different characteristics in terms of neighboring cells and coverage. Cell 1 has a small coverage area that includes the base stations of other cells, whereas cell 2 has a larger coverage area with no neighboring base stations inside this area. We assume also that the C-Cluster has 17 sensors distributed uniformly inside the cluster. We consider two extreme cases of the cluster radius, specifically $5 \%$ and $85 \%$ of the radius of the covering cell.

In Figure 9, we show the distribution of the allowed transmit power in frequency band 2 where the two base stations serving the test cells are idle. The algorithm has different behavior in these two cells when the radius of the cluster change. The transmit power increases with the increase of the radius in the first cell, whereas it decreases in the second. This is due to the fact that the cluster radius in the second cell becomes very large and the contribution of the other cells becomes more important than the covering cell. Moreover, the estimated power decreases when the center of the cluster is in the middle of the tested cell since the sensors's distance to the covering cell becomes higher. It can be noted also that when the radius is $0.38 \mathrm{~km}$ in cell 1 , the C-Ns cannot transmit at all if the center of the cluster is $0.5 \mathrm{~km}$ away from the center of cell 1 . This is due to the fact that all sensors will be under the coverage of the neighboring coverage cell, which is active in frequency band 2 . When the radius of the cluster increases to $1.57 \mathrm{~km}$ some C-Ns will be outside this coverage area and are allowed to transmit. We also evaluated the probability of harmful interference in frequency bands 1 and 2 for all cells and it was significantly low due the conservative approach. 

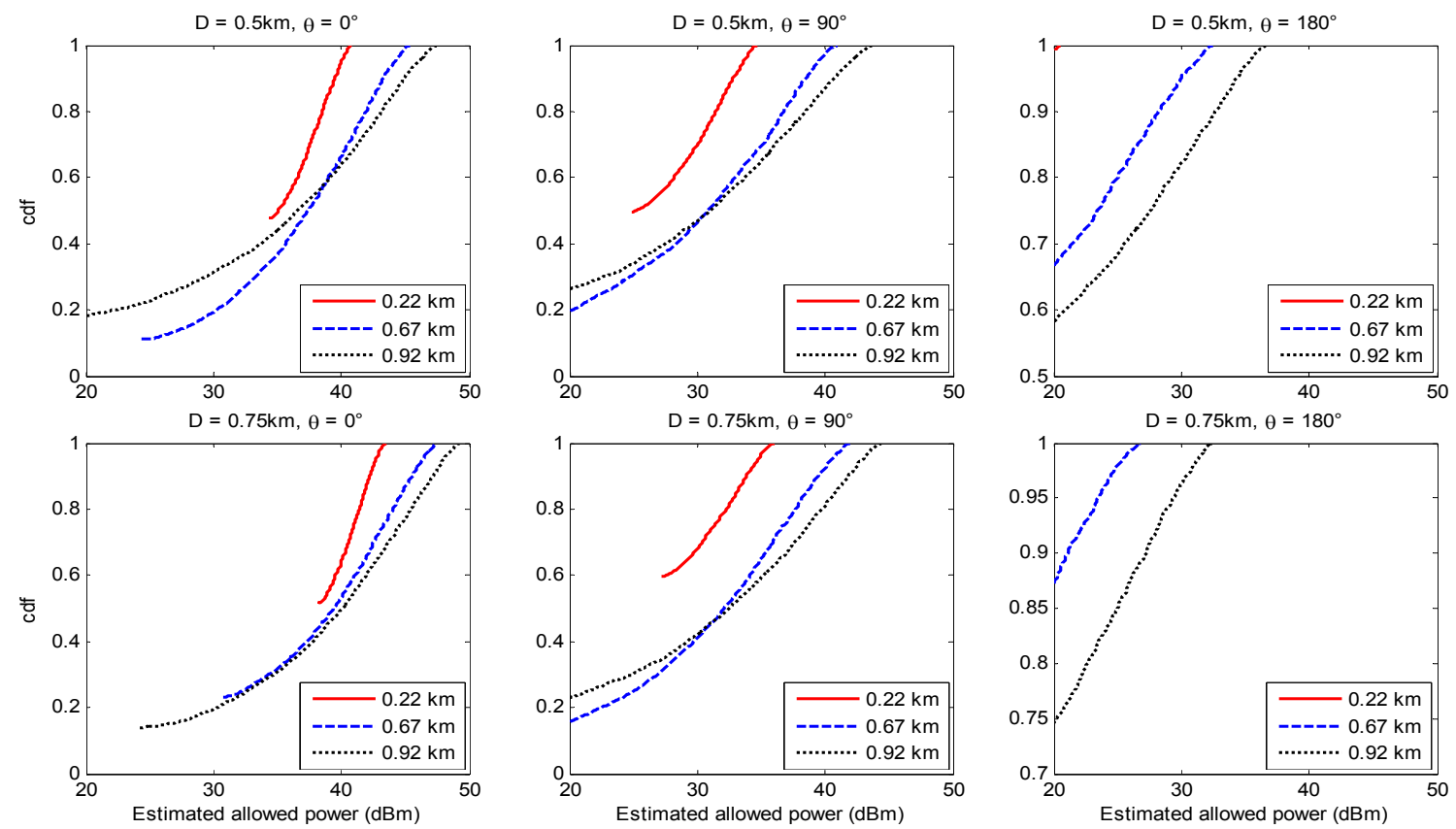

Figure 7: The distribution of the estimated allowed transmit power in scenario 1 for different positions and radii of the S-C (the position and the angle are relative to the idle P-BS and the line joining the two $\mathrm{P}-\mathrm{BSs}$ ).

\section{CONCLUSIONS AND DISCUSSION}

In this paper, we have focused on the problem of opportunistic access when the primary network is a cellular network. In order to enable cognitive radio to use cellular frequency bands, we have assumed that the relative positions of primary base stations are known, which is a realistic assumption in some scenarios. This information is used by a maximum likelihood estimator to determine the closest active cell to the cognitive node, which will experience the highest interference due to cognitive node's activity. Then the cognitive node can estimate its allowed transmit power that satisfies primary constraints.

The proposed approach has been evaluated using simulations from which the following conclusions can be drawn:

- IEEE 802.11-like cognitive radio can opportunistically access cellular frequency bands under some constraints and if the cellular network is willing to share its spectrum.

- Even with the assumption of knowing primary base stations' positions, the detection of spectrum opportunities and the protection of primary users with relatively acceptable probability is only possible under specific constraints. This highlights the fact that without this information, sharing spectrum with cellular network will be very difficult.

- In order to detect base station activity the sensors should be close to the considered base station. This means that a cluster of sensors should only be interested in detection the activity of covering cells.

- The position and the distribution of the sensors can be in most cases more important than the number of these sensors. Hence, developing a model that determines the best position distribution of the sensors remains an open problem.

From these results, we can conclude that the primary network has to do a feasibility study on the possibility of sharing its spectrum and the related conditions to the position, density and distribution of the sensors used by the secondary. The developed model in this paper can be used as a guideline to find these configurations. The main limitation that prevent its implementation in real systems is the assumption of perfect knowledge of propagation models. The knowledge of these models have high impact on both detecting the active cells and estimating the generated interference. Therefore, this limitation should be studied thoroughly, not only in the context of this paper but also for cognitive radio approaches. It can be overcome using different approaches from which we name two based on the knowledge of a family of propagation models adequate for the studied area:

- Use the worst case propagation model. For the detection of the active cells, this would be the one that generates the highest attenuation (i.e, high propagation exponent and shadowing standard deviation), whereas for the estimation of interference the model generating the lowest attenuation is to be used (e.g., free space model). Although it is an easy and feasible solution, it can lead to low performance of the cognitive network.

- Include the different propagation models and their parameters in the MLE problem. This makes the problem more complicated but leads to better performance.

\section{ACKNOWLEDGMENTS}

We acknowledge a partial financial support from European Union through FARAMIR (grant number ICT-248351) and ACROPOLIS (ICT- 257626) projects. We also thank 


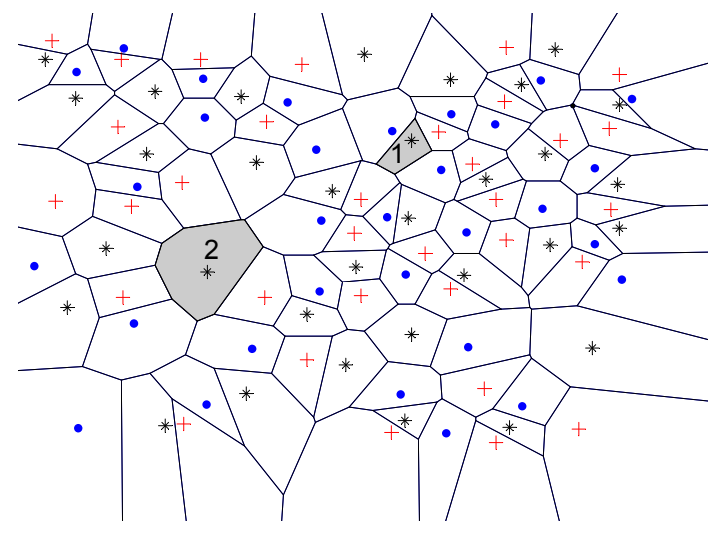

Figure 8: LA scenario where '*', '+' and 'o' refer to cells using frequency bands 1,2 and 3 , respectively. The shaded cells are the cells where the performance of the proposed approach is studied.

the financial support from RWTH Aachen University and the German Research Foundation (Deutsche Forschungsgemeinschaft, DFG) through the UMIC research centre.

\section{REFERENCES}

[1] http://www.t-mobiletowers.com/ [Last visited 02/08/2011].

[2] Second report and order and memorandum opinion and order. in ET Docket No. 04-186 and ET Docket No. 02-380, FCC 08-260, November 42008.

[3] CEPT Report 30: Report from CEPT to the European Commission in response to the Mandate on "the identification of common and minimal (least restrictive) technical conditions for $790-862 \mathrm{MHz}$ for the digital dividend in the European Union", 2009.

[4] 3GPP TR 36.810: "UMTS / LTE in $800 \mathrm{MHz}$ for Europe", March 2010.

[5] I. F. Akyildiz, W.-Y. Lee, M. C. Vuran, and S. Mohanty. Next generation/dynamic spectrum access/cognitive radio wireless networks: a survey. Computer Networks: The International Journal of Computer and Telecommunications Networking, 50(13):2127 - 2159, 2006.

[6] M. M. Buddhikot, P. Kolodzy, S. Miller, K. Ryan, and J. Evans. DIMSUMnet: New directions in wireless networking using coordinated dynamic spectrum access. In the IEEE International Symposium on a World of Wireless, Mobile and Multimedia Networks (WoWMoM 2005), 2005.

[7] K. Harrison and A. Sahai. Potential collapse of whitespaces and the prospect for a universal power rule. In the IEEE International Symposium on New Frontiers in Dynamic Spectrum Access Networks (DySPAN 2011), 2011.

[8] S. Haykin. Cognitive radio: brain-empowered wireless communications. IEEE Journal on Selected Areas in Communications, 23(2):201-220, Feb. 2005.

[9] N. Hoven and A. Sahai. Power scaling for cognitive radio. In the International Conference on Wireless Networks, Communications and Mobile Computing, volume 1, pages 250-255, 2005.
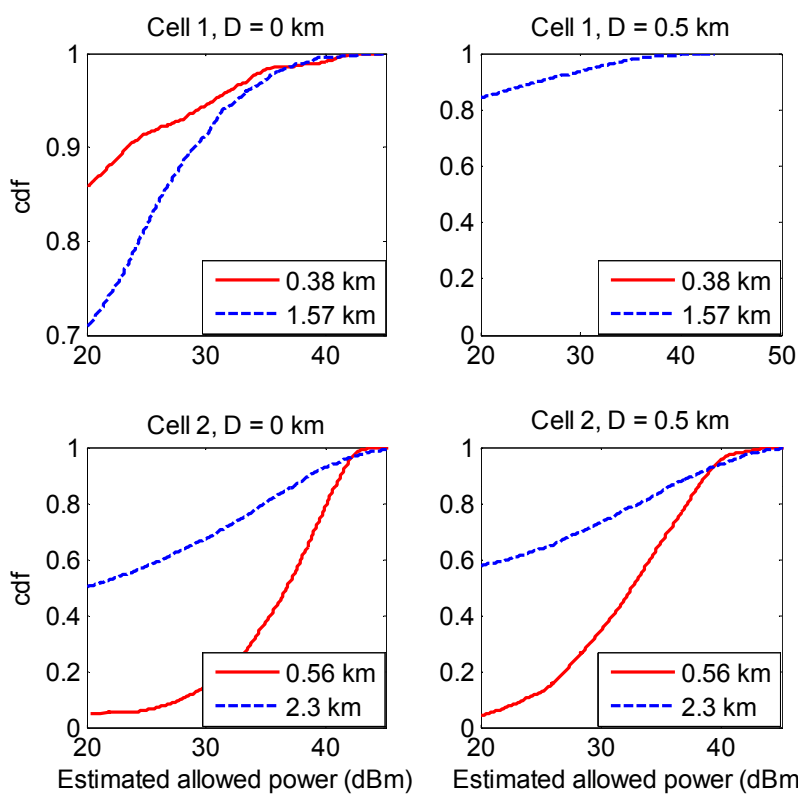

Figure 9: The distribution of the estimated transmit power in the LA scenario when the two base stations are idle for different positions and size of the cluster.

[10] L. Maciel, H. Bertoni, and H. Xia. Unified approach to prediction of propagation over buildings for all ranges of base station antenna height. IEEE transactions on vehicular technology, vol. 42(no. 1):41-45, 1993.

[11] N. Miliou, A. Moustakas, and A. Polydoros. Interference source localization and transmit power estimation under log-normal shadowing. In the 2011 European Signal Processing Conference (Eusipco 2011), 2011.

[12] R. Murty, R. Chandra, T. Moscibroda, and V. Bahl. Senseless: A database-driven white spaces network. In the IEEE International Symposium on New Frontiers in Dynamic Spectrum Access Networks (DySPAN 2011), 2011.

[13] A. O. Nasif and B. L. Mark. Opportunistic spectrum sharing with multiple cochannel primary transmitters. IEEE Transactions on Wireless Communications, 8(11):5702 - 5710, 2009.

[14] J. Nasreddine, A. Achtzehn, J. Riihijärvi, and P. Mähönen. Enabling secondary access through robust primary user channel assignment. In IEEE Global Telecommunications Conference (GLOBECOM 2010), 2010.

[15] J. Nasreddine, J. Riihijärvi, and P. Mähönen. Location-based adaptive detection threshold for dynamic spectrum access. In the IEEE International Symposium on New Frontiers in Dynamic Spectrum Access Networks (DySPAN 2010), 2010.

[16] J. Nasreddine, O. Sallent, J. Pérez-Romero, and R. Agustí. Positioning-based framework for secondary spectrum usage. Physical Communication, 1(2):121-133, 2008 .

[17] R. Tandra, M. Mishra, and A. Sahai. What is a spectrum hole and what does it take to recognize one? Proceedings of the IEEE, 97(5):824-848, 2009. 